\title{
Interactive comment on "Surface-circulation change in the Southern Ocean across the Middle Eocene Climatic Optimum: inferences from dinoflagellate cysts and biomarker paleothermometry" by Margot J. Cramwinckel et al.
}

Margot J. Cramwinckel et al.

m.j.cramwinckel@uu.nl

Received and published: 15 August 2019

Please see the attached supplementary pdf for our author response. 
Interactive comment on Clim. Past Discuss., https://doi.org/10.5194/cp-2019-35, 2019.

CPD

Interactive comment 